\section{Leishmaniose tegumentar americana canina em municípios do norte do Estado do Paraná, Brasil}

\author{
Canine American cutaneous leishmaniasis \\ in municipalities of northern Paraná State, Brazil
}

Paulo Donizeti Zanzarini 1 Demilson Rodrigues dos Santos 2 Ademar Rodrigues dos Santos 2 Otílio de Oliveira ${ }^{2}$

Luiz Paschoal Poiani 2 Maria Valdrinez Campana Lonardoni 1 Ueslei Teodoro ${ }^{1}$

Thaís Gomes Verzignassi Silveira ${ }^{1}$

\section{Introdução}

American cutaneous leishmaniasis (ACL) is an endemic disease in northern Paraná State, where it affects humans and dogs. This study aimed to verify the occurrence of the canine disease in ACL endemic areas. From September 1999 to July 2002, dogs were investigated in 7 rural areas of 5 municipalities where autochthonous human cases of ACL had been reported. Parasitological and serological methods were used. Fourteen of 67 dogs studied (20.9\%) had lesions suggestive of ACL, of which 3 (21.4\%) were infected with Leishmania sp. Indirect immunofluorescence for Leishmania (Viannia) braziliensis was positive in 37 (55.2\%) of 67 dogs. The results showed that human and canine ACL occur simultaneously and indicate the need for additional studies to elucidate the role of dogs in the ACL transmission cycle in the area studied.

Endemic Diseases; Zoonoses; Leishmaniasis
A leishmaniose tegumentar americana (LTA) é amplamente distribuída no Brasil, ocorrendo em todos os Estados, tem caráter endêmico no Paraná 1, com notificação em 276 dos 399 municípios, principalmente no norte e oeste deste Estado 2.

A LTA é uma zoonose originalmente silvestre, mas vem ocorrendo em áreas rurais e urbanas, sendo freqüente o encontro de animais domésticos (cães e eqüídeos) infectados 3,4,5. No noroeste do Paraná a presença de cães infectados por Leishmania (Viannia) braziliensis 6,7 e com sorologia positiva para LTA 8 tem sido relatada em áreas de ocorrência da doença humana, onde há intensa exploração agrícola e pecuária 2 .

Como a transmissão da LTA tem aumentado no ambiente doméstico e há registros de altas taxas de infecção em cães, cresce a suspeita de que esses animais possam atuar como reservatórios de Leishmania sp. 9. Vexenat et al. 10 verificaram que Lutzomyia whitmani infectava-se com L. braziliensis quando alimentados em lesões ulceradas, mas não quando alimentados em áreas não ulceradas da pele de cães infectados. Pirmez et al. 11 não conseguiram isolar o parasito da pele normal de cães e Rangel et al. 12 mostraram que flebotomíneos alimentavam-se menos freqüentemente em humanos e cães que em eqüídeos. Contudo, o 
papel do cão no ciclo doméstico de transmissão da LTA permanece polêmico 9,11,13.

Este trabalho teve por objetivo investigar a LTA canina em localidades rurais onde ocorreram casos de LTA humana, em área endêmica no norte do Estado do Paraná, Brasil.

\section{Materiais e métodos}

O estudo foi realizado no período de setembro de 1999 a julho de 2002, em sete localidades rurais, nos municípios de Lobato $\left(23^{\circ} 00^{\prime} 30^{\prime \prime} \mathrm{S}\right.$ e $\left.51^{\circ} 54^{\prime} 00^{\prime \prime} \mathrm{W}\right)$, Maringá $\left(23^{\circ} 25^{\prime} 00^{\prime \prime} \mathrm{S}\right.$ e $51^{\circ} 25^{\prime}$ $00^{\prime} \mathrm{W}$ ), Colorado ( $22^{\circ} 50^{\prime} 00^{\prime \prime} \mathrm{S}$ e $\left.51^{\circ} 53^{\prime} 00^{\prime \prime} \mathrm{W}\right)$, Santa Fé $\left(23^{\circ} 02^{\prime} 15^{\prime \prime} \mathrm{S}\right.$ e $\left.51^{\circ} 48^{\prime} 19^{\prime \prime} \mathrm{W}\right)$ e Mandaguari $\left(23^{\circ} 31^{\prime} 00^{\prime}\right.$ S e $\left.51^{\circ} 41^{\prime} 00^{\prime \prime} \mathrm{W}\right)$ pertencentes à mesorregião norte central paranaense, de clima subtropical úmido mesotérmico (Figura 1). Nestas localidades ocorreram 22 casos autóctones de LTA humana, diagnosticados pela pesquisa direta do parasito, reação de imunofluorescência indireta e a intradermorreação de Montenegro.

Os 67 cães estudados foram examinados quanto à presença de lesão e de todos foi coletado uma amostra de sangue. Dos cães que apresentavam lesão foi obtido material por biópsia ou escarificação de borda interna de lesão para pesquisa direta do parasito. Os esfregaços de material de lesão foram corados pelo método de Giemsa e analisados por microscopia para a pesquisa de formas amastigotas de Leishmania sp.

Para a reação de imunofluorescência indireta (IFI) utilizaram-se formas promastigotas de $L$. (V.) braziliensis e conjugado anti-IgG canina-fluoresceína (Sigma). Títulos $\geq 40$ foram considerados significativos 8 . A IFI para doença de Chagas foi realizada em todos os cães com IFI positiva para LTA. Utilizou-se antígeno Imunocruzi (Biolab). Foram considerados positivos para doença de Chagas os soros que apresentaram títulos $\geq 80$ e quando havia diferença de pelo menos duas diluições entre os re-
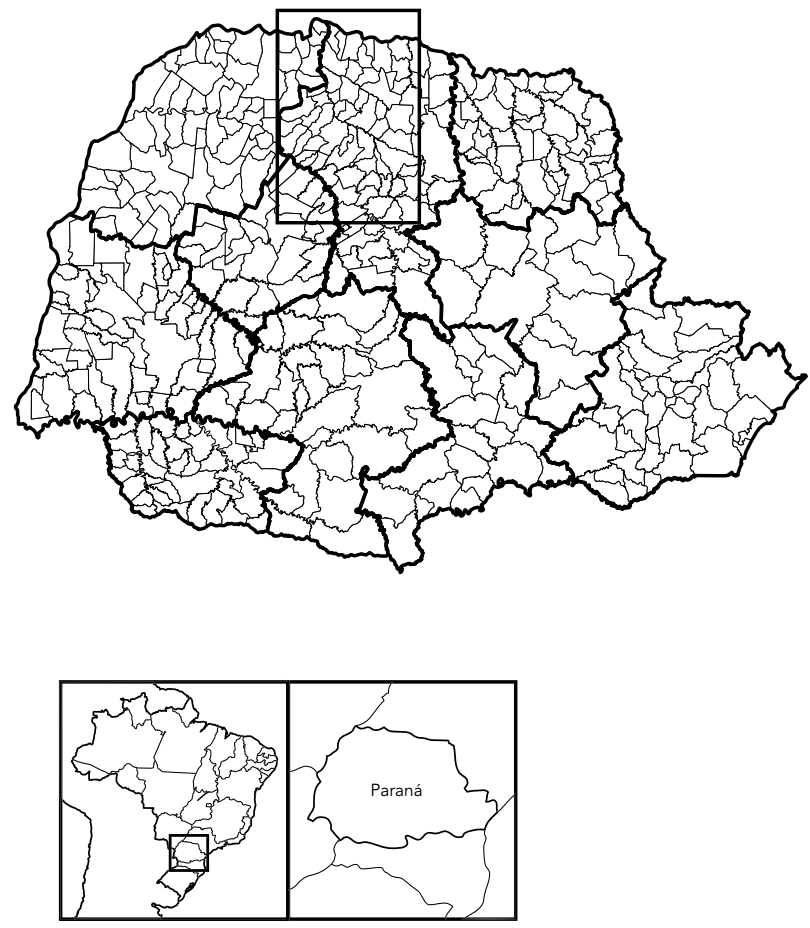

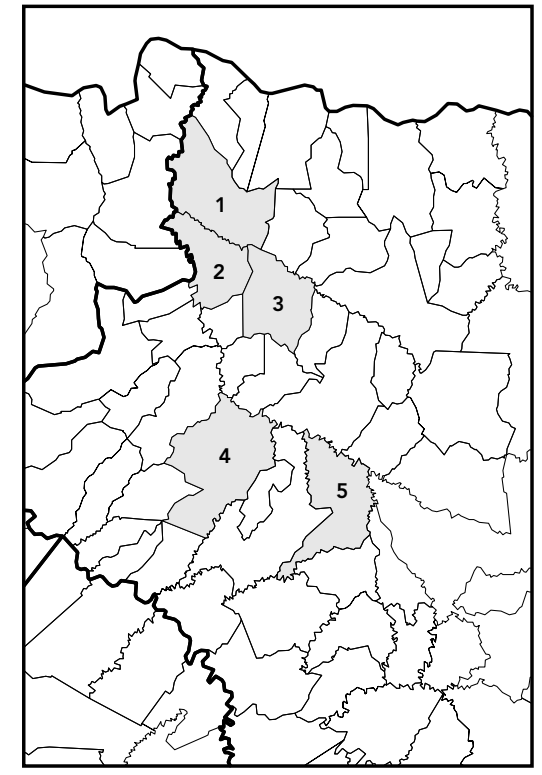

1 - Colorado 2 - Lobato 3 - Santa Fé 4 - Maringá 5 - Mandaguari 
sultados da IFI para doença de Chagas e da IFI para leishmaniose.

\section{Resultados}

Nas sete localidades onde ocorreram casos humanos autóctones de LTA foram investigados 67 cães. Os resultados do diagnóstico laboratorial para LTA dos cães encontram-se na Tabela 1 .

Nessas localidades aconteceram 22 casos humanos, sendo sete mulheres e 15 homens. Todos os casos humanos foram investigados laboratorialmente e apresentaram pelo menos um dos testes positivo para LTA. Em todas as localidades, a investigação dos cães foi realizada após a constatação dos casos humanos de LTA.

Dos 67 cães estudados, 14 (20,9\%) apresentavam lesões sugestivas de LTA, dos quais 3 $(21,4 \%)$ tiveram a pesquisa direta do parasito em lesão positiva e a IFI positiva. Dos 11 cães com lesão, $9(81,18 \%)$ tiveram IFI positiva. Cinqüenta e três cães não apresentavam lesão, no entanto, 25 (47,2\%) deles tiveram IFI positiva para LTA. Um cão da localidade Sítios Reunidos (Maringá) foi considerado positivo para doença de Chagas.

Dos três cães com pesquisa direta positiva em lesão, dois residiam no Município de Lobato (Fazenda da Barra e Fazenda Remanso) e outro no Município de Maringá (São Domingos).
Em seis das sete localidades estudadas foram detectados cães com lesão sugestiva de LTA. No entanto, em todas as localidades foram encontrados cães sem lesão, mas com a sorologia positiva.

\section{Discussão}

No Estado do Paraná a ocorrência de LTA está associada à $L .(V$.) braziliensis 1 . No norte do Paraná a presença de cães com infecção por $L$. braziliensis 6,7 tem sido constatada em áreas rurais onde a LTA se mantém endêmica e associada aos ambientes alterados 2, que favorecem a adaptação de espécies de flebotomíneos 14. Na maioria das localidades no norte do $\mathrm{Pa}-$ raná as espécies de flebotomíneos predominantes são $N$. neivai ou $N$. whitmani, com número sempre elevado no domicílio e, especialmente, no peridomicílio 14 . Destaque-se que $N$. whitmani já foi encontrada infectada por $L$. $(V$.$) braziliensis no Estado do Paraná 15$.

Este estudo foi realizado em localidades rurais onde haviam sido constatados casos humanos autóctones de LTA. A investigação dos cães residentes nessas localidades mostrou que 14 animais $(20,9 \%)$ apresentavam lesão, dos quais três $(21,4 \%)$ foram positivos na pesquisa direta do parasito de Leishmania sp. e na IFI. Nove $(81,8 \%)$ dos 11 cães com lesão e pes-

Tabela 1

Resultados do diagnóstico para leishmaniose tegumentar americana em cães de localidades rurais

de municípios do norte do Estado do Paraná, Brasil, onde ocorreram casos humanos, no período

de setembro de 1999 a julho de 2002.

\begin{tabular}{|c|c|c|c|c|c|c|}
\hline \multirow[t]{2}{*}{ Município } & \multirow[t]{2}{*}{ Localidade } & \multicolumn{2}{|c|}{ Humanos } & \multicolumn{3}{|c|}{ Cães } \\
\hline & & $\mathrm{n}$ & Sexo (F:M) & $\mathrm{n}$ & Pesquisa direta* & $|F|^{*}$ \\
\hline \multirow[t]{2}{*}{ Lobato } & Fazenda da Barra & 5 & $3: 2$ & 4 & $1 / 2$ & $4 / 4$ \\
\hline & Fazenda Remanso & 5 & $0: 5$ & 3 & $1 / 1$ & $1 / 3$ \\
\hline \multirow[t]{2}{*}{ Maringá } & São Domingos & 4 & $3: 1$ & 13 & $1 / 4$ & $12 / 13$ \\
\hline & Sítios Reunidos & 1 & $0: 1$ & 12 & $0 / 4$ & $7 * \star / 12$ \\
\hline Santa Fé & Água do O & 2 & $1: 1$ & 14 & $0 / 2$ & $4 / 14$ \\
\hline Colorado & Água do Cedro & 2 & $0: 2$ & 5 & $0 / 0$ & $2 / 5$ \\
\hline Mandaguari & $\begin{array}{l}\text { Sítios São José } \\
\text { e Lombo da Égua }\end{array}$ & 3 & $0: 3$ & 16 & $0 / 1$ & $8 / 16$ \\
\hline Total & & 22 & $7: 15$ & 67 & $3 / 14$ & $38 / 67$ \\
\hline
\end{tabular}

IFI = imunofluorescência indireta.

* Número de positivos/número de analisados.

** Um cão com sorologia positiva para doença de Chagas. 
quisa direta do parasito negativa, apresentaram IFI positiva, demonstrando que apesar de não ter sido encontrado parasito nas lesões, esses animais poderiam estar infectados por Leishmania sp. A baixa positividade da pesquisa direta do parasito pode ser conseqüência da escassez de parasitos nas lesões de cães, como constatado por Marco et al. 16. Desta forma, o diagnóstico da LTA canina deve levar em consideração a eventual presença de quadros dermatológicos incomuns, a dificuldade de encontro do parasito e a possível variação na resposta imune individual. Por isso, em cães com lesões sugestivas, provenientes de áreas endêmicas, Madeira et al. 4 sugerem que seja realizado o acompanhamento periódico com o objetivo de confirmar o diagnóstico da LTA.

Observou-se que dos 53 cães que não apresentavam lesão, $25(47,2 \%)$ tiveram a IFI positiva para LTA. A presença de cães sem lesão e com sorologia positiva em áreas endêmicas tem sido reportada por outros autores 5,11,17,18. Em inquérito sorológico realizado nos municípios de Jussara e Terra Boa, região noroeste do Estado do Paraná, Silveira et al. 8 encontraram $18,2 \%$ dos cães com sorologia positiva. Barbosa et al. 19 consideraram como infectados aqueles cães que foram reagentes a pelo menos uma das técnicas sorológicas utilizadas (ELISA e IFI), assumindo que a inexistência de lesões suspeitas em cães de áreas endêmicas de LTA humana não significa ausência da infecção canina. A identificação freqüente de cães domésticos com a infecção por Leishmania sp., associada à presença da doença humana, sinaliza para o envolvimento desses animais no ciclo de transmissão desses parasitos 20 .

Falqueto et al. 13 observaram nítida relação entre a presença de cães infectados e a ocorrência de novos casos humanos da doença e pro- puseram que a doença estivesse sendo mantida pelos cães domésticos, corroborando as observações de Oliveira-Neto et al. ${ }^{3}$, de que os casos caninos de LTA antecedem a ocorrência dos casos humanos. Neste estudo não foi possível estabelecer se a doença canina antecedeu a humana, pois os cães foram investigados em localidades onde já haviam sido diagnosticados os casos humanos. Porém, foi interessante notar que em seis das sete localidades estudadas foram detectados cães com lesão sugestiva de LTA e que em todas elas foram encontrados animais sem lesão, mas com sorologia positiva.

A ocorrência da LTA em humanos e cães em áreas onde a LTA é endêmica suscita questionamentos quanto ao papel do cão no ciclo de transmissão do parasito. Reithinger et al. 9 sugerem que seja investigada a presença do parasito em vetores que entram em contato com os cães e os animais silvestres que freqüentam o ambiente peridomiciliar, bem como a persistência da infecção em cães e sua possível relação com a infecção humana.

Os resultados deste trabalho mostram que na região estudada a LTA humana e canina ocorrem simultaneamente, o que já foi relatado em outras áreas endêmicas de LTA. A presença de cães com infecção por Leishmania sp., associada à sorologia positiva mostra que o parasito está circulando nas áreas onde o estudo foi realizado. Contudo, nas regiões endêmicas de LTA no Estado do Paraná, ainda não está claro se o cão tem um papel na manutenção do parasito ou se a infecção é casual. Futuros estudos sobre a infecção em cães com sorologia positiva, utilizando técnicas mais sensíveis, bem como o acompanhamento desses animais e a investigação da infecção em vetores poderão ajudar a esclarecer o papel do cão no ciclo de transmissão do parasito.

\section{Resumo}

A leishmaniose tegumentar americana (LTA) é endêmica no norte do Estado do Paraná, Brasil, afetando tanto o homem como os cães. O objetivo deste trabalho foi estudar a ocorrência da doença na população canina em áreas endêmicas de LTA. No período de setembro de 1999 a julho de 2002, foram investigados cães em sete localidades rurais de cinco municípios onde ocorreram casos humanos autóctones da doença, utilizando métodos parasitológicos e sorológicos. Dos 67 cães estudados, 14 (20,9\%) tinham lesões sugestivas de LTA, dos quais $3(21,4 \%)$ estavam infectados por Leishmania sp. Trinta e sete $(55,2 \%)$ cães apresentaram imunofluorescência indireta positiva para Leishmania (Viannia) braziliensis. Os resultados mostram a ocorrência simultânea de leishmaniose humana e canina e indicam a necessidade de estudos adicionais para esclarecer o papel do cão no ciclo de transmissão do parasito nessas áreas.

Doenças Endêmicas; Zoonoses; Leishmaniose 


\section{Colaboradores}

P. D. Zanzarini contribuiu na realização dos ensaios em laboratório, na análise de dados, na elaboração do manuscrito e na aprovação final do texto. D. R. Santos, A. R. Santos, O. Oliveira e L. P. Poiani contribuíram no delineamento do trabalho e na aquisição e análise de dados. M. V. C. Lonardoni e U. Teodoro contribuí-

\section{Referências}

1. Silveira TGV, Arraes SMAA, Bertolini DA, Teodoro U, Lonardoni MVC, Roberto ACBS, et al. Observações sobre o diagnóstico laboratorial e a epidemiologia da leishmaniose tegumentar americana no Estado do Paraná, Sul do Brasil. Rev Soc Bras Med Trop 1999; 32:413-23.

2. Lima AP, Minelli L, Comunello E, Teodoro U. Distribuição da leishmaniose tegumentar por imagens de sensoriamento remoto orbital, no Estado do Paraná, Sul do Brasil. An Bras Dermatol 2002; 77:681-92.

3. Oliveira-Neto MP, Pirmez C, Rangel E, Schubach A, Grimaldi Jr. G. An outbreak of American Cutaneous Leishmaniasis (Leishmania braziliensis braziliensis) in a periurban area of Rio de Janeiro City, Brazil: clinical and epidemiological studies. Mem Inst Osvaldo Cruz 1988; 83:427-35.

4. Madeira MF, Uchoa CMA, Leal CA, Silva RMM, Duarte R, Magalhães CM, et al. Leishmania (Viannia) braziliensis em cães naturalmente infectados. Rev Soc Bras Med Trop 2003; 36:551-5.

5. Santos GPL, Sanavria A, Marzochi MCA, Santos EGOB, Silva VL, Pacheco RS, et al. Prevalência da infecção canina em áreas endêmicas de leishmaniose tegumentar americana, do Município de Paracambi, Estado do Rio de Janeiro, no período entre 1992 e 1993. Rev Soc Bras Med Trop 2005; 38:161-6.

6. Lonardoni MVC, Teodoro U, Arraes SMAA, Silveira TGV, Bertolini DA, Ishikawa EAY, et al. Nota sobre leishmaniose canina no noroeste do Estado do Paraná, Sul do Brasil. Rev Saúde Pública 1993; 27:378-9.

7. Velasquez LG, Membrive N, Membrive U, Rodrigues G, Reis N, Lonardoni MVC, et al. Aplicação da reação em cadeia da polimerase (PCR) na investigação da leishmaniose tegumentar em cães na Região Noroeste do Estado do Paraná. Rev Bras Anal Clin 2004; 36:50B.

8. Silveira TGV, Teodoro U, Lonardoni MVC, Toledo MJO, Bertolini DA, Arraes SMAA, et al. Investigação sorológica em cães de área endêmica de leishmaniose tegumentar, no Estado do Paraná, Sul do Brasil. Cad Saúde Pública 1996; 12:89-93.

9. Reithinger R, Davies CR. Is the domestic dog (Canis familiaris) a reservoir host of American cutaneous leishmaniasis? A critical review of the current evidence. Am J Trop Med Hyg 1999; 61:530-41.

10. Vexenat JA, Barreto AC, Rosa AC. Experimental infection of Lutzomya whitmani in dog infected with Leishmania braziliensis braziliensis. Mem Inst Oswaldo Cruz 1986; 81:125-6. ram na análise dos dados, na elaboração do manuscrito e na revisão e aprovação da versão final do texto. T. G. V. Silveira orientou a realização do trabalho, contribuiu na análise dos dados, na elaboração do manuscrito e na revisão e aprovação final do texto.

11. Pirmez C, Coutinho SG, Marzochi MCA, Nunes MP, Grimaldi Jr. G. Canine American cutaneous leishmaniasis: a clinical and immunological study in dogs naturally infected with Leishmania braziliensis in an endemic area of Rio de Janeiro, Brazil. Am J Trop Med Hyg 1988; 38:52-8.

12. Rangel EF, Azevedo AC, Andrade CA, Souza NA, Wermelinger ED. Studies on sandfly fauna (Diptera: Psychodidae) in a foci of cutaneous leishmaniasis in Mesquita, Rio de Janeiro State, Brazil. Mem Inst Oswaldo Cruz 1990; 85:39-45.

13. Falqueto A, Coura JR, Barros GC, Grimaldi G, Sessa PA, Daher Carias VR, et al. Participação do cão no ciclo de transmissão da leishmamiose tegumentar no Município de Viana, Estado do Espírito Santo, Brasil. Mem Inst Oswaldo Cruz 1986; 81:155-63.

14. Membrive NA, Rodrigues G, Membrive U, Monteiro WM, Neitzke HC, Lonardoni MVC, et al. Flebotomíneos de municípios do Norte do Estado do Paraná, sul do Brasil. Entomologia y Vectores 2004; 11:673-80.

15. Luz E, Membrive N, Castro EA, Dereure J, Pratlong F, Dedet JA, et al. Lutzomya whitmani (Díptera: Psychodidade) as vector of Leishmania (V.) braziliensis in Paraná State, southern Brasil. Ann trop Med Parasitol 2000; 94:623-31.

16. Marco JD, Padilha AM, Diosque P, Fernandez MM, Malchiodi EL, Basombrio MA. Force of infection and evolution of lesions of canine tegumentary leishmaniasis in Northwestern Argentina. Mem Inst Oswaldo Cruz 2001; 96:649-52.

17. Marzochi MVC, Barbosa-Santos EGO. Evaluation of a skin test on the canine mucocutaneous leishmaniasis diagnosis. Mem Inst Oswaldo Cruz 1988; 83:391-2.

18. Madeira MF, Serra CMB, Uchoa CMA, Duarte R, Cruz DAM, Perdomo CC. Leishmaniose canina: avaliação sorológica de 310 cães na região de Itaipu, Rio de Janeiro. Cad Saúde Pública 2000; 16:568.

19. Barbosa GMS, Marzochi MCA, Massard CL, Lima GPS, Confort EM. Aspectos epidemiológicos da leishmaniose tegumentar americana em cães, no Município de Paraty, Estado do Rio de Janeiro, Brasil. Cad Saúde Pública 1999; 15:641-6.

20. Lopes UG, Momem H, Grimaldi Jr. G, Marzochi MCA, Pacheco RS, Morel CM. Schizodeme and zymodeme characterization of Leishmania in the investigation of foci visceral and cutaneous leishmaniasis. J Parasitol 1984; 70:89-98.

Recebido em 14/Out/2004

Versão final reapresentada em 18/Ago/2005

Aprovado em 22/Ago/2005 